\title{
Nonalcoholic-Fatty-Liver-Disease and Nonalcoholic Steatohepatitis: Successful Development of Pharmacological Treatment Will Depend on Translational Research
}

\author{
Aline Gottlieb Wesal Mosthael Jan-Peter Sowa Ali Canbay \\ Department of Gastroenterology, Hepatology, and Infectious Diseases, Otto-von-Guericke University Magdeburg, \\ Magdeburg, Germany
}

\section{Keywords}

Nonalcoholic steatohepatitis · Clinical trials · Fibrosis .

Hepatitis · Steatosis

\begin{abstract}
Background: Nonalcoholic-fatty-liver-disease/nonalcoholic steatohepatitis (NAFLD/NASH) is expected to become the leading liver disease worldwide. Typical liver-related complications are fibrosis, cirrhosis, and the development of hepatocellular cancer (HCC) with the need for liver transplantation. Up to now there is no approved pharmacotherapy. Indeed, this might be due to the complexity of this disease. While the cheapest therapeutic approach is still a lifestyle change leading to weight loss, the proportion of people achieving sufficient weight reduction without additional support is low. Newly developed drugs are expensive and lack a breakthrough in therapeutic success. One reason might be that drugs developed often derive from murine models. Unfortunately, there is little overlap between genes in human and mice that are responsible for the development of NAFLD/NASH. This review aims at summarizing latest developments as well as stress again that more translational research is necessary. Summary: Therapy of NAFLD/NASH is
\end{abstract}

\section{KARGER}

() 2018 S. Karger AG, Basel

E-Mail karger@karger.com

www.karger.com/dig easy and very complex at the same time, as the current main target is weight reduction. Since this is in fact not easily achieved and maintained by many affected individuals, pharmacotherapy to halt the progression of NAFLD/NASH is urgently warranted. More translational studies are needed to understand the metabolic mechanisms and interactions between the liver, gut, oxidative stress and the processes leading to NAFLD progression and HCC development, even in the absence of cirrhosis.

(c) 2018 S. Karger AG, Basel

\section{Diagnostic Possibilities in Nonalcoholic-Fatty-Liver- Disease/Nonalcoholic Steatohepatitis}

Over the last couple of years, more systematic approaches have been developed to find people at risk of nonalcoholic fatty liver disease (NALFD) or nonalcoholic steatohepatitis (NASH). Risk factors include type 2 diabetes (T2DM), obesity, hypertension, and dyslipidemia with or without elevated liver enzymes. When NAFLD/ NASH is suspected, further measurements are necessary such as liver stiffness (fibroscan ${ }^{\circledR}$ ), CT-scan (sensitive and specific, but exposes the patient to radiation), or

Prof. Dr. med. A. Canbay

Department of Gastroenterology, Hepatology, and Infectious Diseases Otto-von-Guericke University Magdeburg

Leipziger Street 44, DE-39120 Magdeburg (Germany)

E-Mail Ali.canbay@med.ovgu.de 
MRI, which is the gold standard, but currently the most expensive method to identify liver steatosis. Currently, the only way to reliably diagnose steatohepatitis (NASH) is a liver biopsy with histological evaluation. Its presence and the fibrotic stage of the liver are the best predictors of cirrhosis [1]. MR elastography is the gold standard for detection of fibrosis but often not available or not tolerated by patients due to duration of the examination and claustrophobia. Noninvasive tests for the assessment of hepatic fibrosis, such as the fibrosis- 4 index or the aminotransferase (AST) to platelet ratio (APRI), have a high negative predictive value $[2,3]$, which excludes fibrosis with high accuracy. Unfortunately, their ability to diagnose cirrhosis is limited. Specific laboratory tests have not convincingly shown to be superior to simple laboratory analysis and a relevant number of unclear cases still require liver biopsy.

\section{Genetic Background in NAFLD}

There is evidence linking NAFLD to a genetic background in some cases. Leading SNP polymorphisms that have been associated with NAFLD and severity of NAFLD in human studies are PNPLA3 and TM6SF2. The actual role of the genetic background and its importance on progression of NAFLD/NASH are yet to be determined. Thus, common screening for these polymorphisms is not recommended yet [4]. These genetic polymorphisms can play a role in patients with lean NAFLD. Data available so far are still limited [5]. In case of patients dealing with a lean NAFLD, other factors such as celiac disease (as a symptom of gut leakage), lysosomal acid lipase deficiency (LAL-D) and Gaucher's disease should be excluded, aside the genetic testing for NAFLD. Moreover, while individuals might not be obese $\left(>30 \mathrm{~kg} / \mathrm{m}^{2}\right)$ or not even overweight $\left(>25 \mathrm{~kg} / \mathrm{m}^{2}\right)$ by BMI they might still have abdominal obesity (waist circumference $>80 \mathrm{~cm}$ in women, $>94$ $\mathrm{cm}$ in men), which still confers a significant metabolic risk on the liver. A specific increase of the visceral adipose tissue depot might be the basis of "lean" NAFLD and should be examined in greater detail in future studies.

\section{Conservative and Pharmacological Treatment of NAFLD}

NAFLD and NASH are - despite the simple underlying cause of excess adipose tissue mass - highly complex diseases with incompletely understood mechanisms of devel- opment and progression. So far it has been very difficult to develop a successful drug targeting NAFLD. One of the reasons for this is that it is a highly complex disease caused by multiple factors. Another very important reason has been discovered in a recent study: most of the drugs targeting NAFLD are being tested in murine models. But there is only very little overlap between murine and human transcriptome in NAFLD or NASH with only between 1 and 18 significantly and concordantly regulated genes out of 65 (NAFLD) and 177 (NASH) genes regulated in humans [6]. This clearly shows that the mechanisms of human and murine NAFLD and NASH differ substantially. With a different genetic background, but also profound anatomical differences regarding the digestive system, it is necessary to move away from murine models and perform more human studies in order to target NAFLD.

Nevertheless, on an empirical basis, there are 3 pillars for treatment regimens for NAFLD or NASH (Fig. 1). These comprise weight loss either by lifestyle changes or surgical approaches, targeting the insulin resistance of the liver and systemically, and targeting factors affecting the liver itself.

\section{Weight Loss/Lifestyle Changes}

Some studies have been conducted, showing that weight loss in combination with a diet and physical activity is able to improve steatosis of the liver. For example, Vilar-Gomez et al. [7] studied paired liver biopsies (weeks 1 and 52) of 261 patients that changed their life style for a year. This included a reduction of their daily calorie intake by $750 \mathrm{kcal} /$ day as well as the encouragement to walk 200 min per week. Patients who were able to reduce their body weight by more than $10 \%$ achieved reduction in scores for steatosis, ballooning, and inflammation. Some even showed a decrease in the fibrosis stage. Participants who were able to reduce their body weight between 7 and $10 \%$ showed an improvement of histological features but no improvement in the fibrotic stage. If the weight loss was below $5 \%$, no significant improvement could be seen after a year. Moreover, behavioral therapy seems to be helpful and very cost effective. It is important to note that it is still unclear whether the effect on the liver derives from the weight loss alone or alterations in nutrient uptake. Nevertheless, for most patients, weight loss by intense lifestyle change should be the central therapy.

\section{Targeting Dyslipidemia and Insulin Resistance}

Statins

Statins are safe in NASH [8] and are associated with a reduced mortality [9]. There is strong evidence that statins have an anti-inflammatory effect in the body and
Gottlieb/Mosthael/Sowa/Canbay 
Fig. 1. Therapeutic options for NAFLD according to their drug-dependency and costs. While conservative weight loss regimens are rather cost effective, they rely on the motivation of the patient. Pharmaceutical options are more costly and have to be considered experimental in most cases when applied for NAFLD. * Bariatric surgery is an effective treatment covering all aspects: weight loss, insulin resistance, and liver-specific outcomes. But bariatric surgery is costly and requires intense post-surgical supervision as well as additional treatment if necessary.

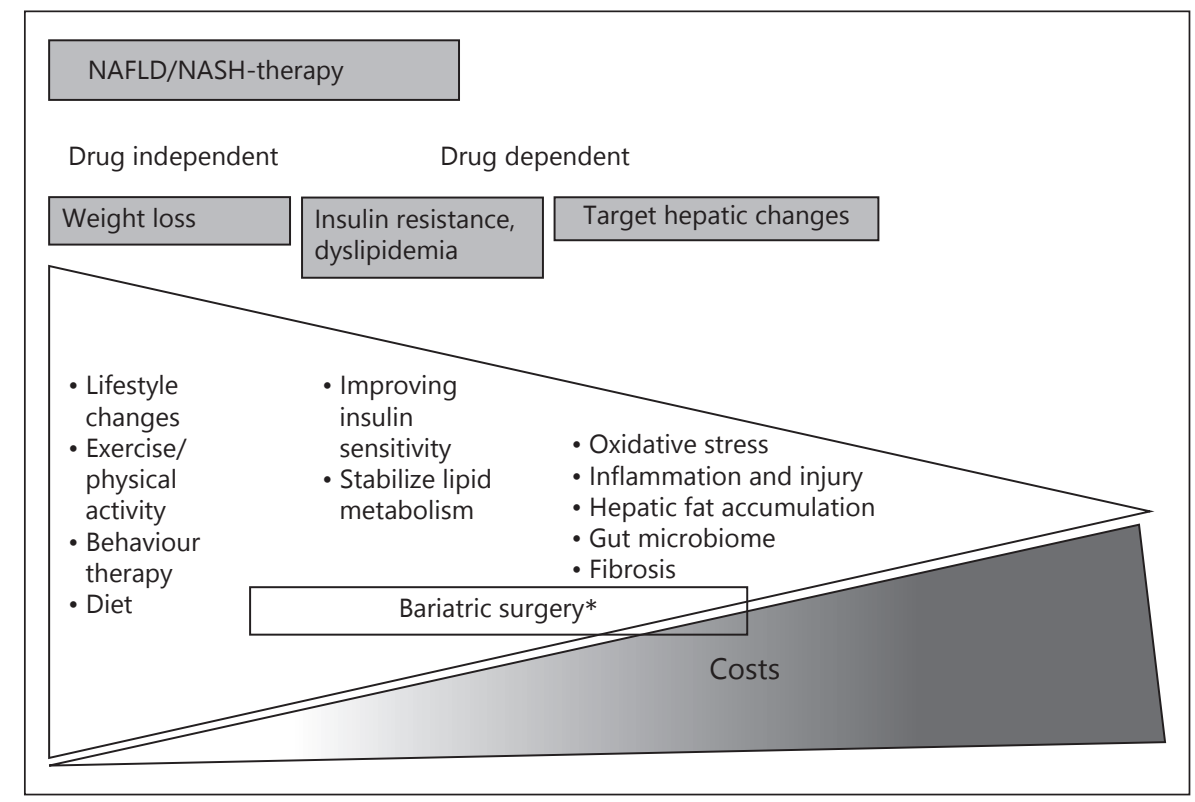

DPP4 Inhibitors

Sitagliptin is an example of a DDP-4 inhibitor. A Phase IIa trial in NAFLD has been completed recently and the substance was approved for T2DM treatment. It has been shown that hepatocyte ballooning and NAS in diabetic patients with NASH improved significantly under therapy [20].

\section{Metformin}

Metformin is the first-line agent for T2DM. Moreover, it is able to reduce the risk of all diabetes-related end points including myocardial infarction, large vessel and microvascular disease as well as cardiovascular mortality [12]. Although, studies have not demonstrated an improvement in liver enzymes and liver histology due to Metformin treatment, it is still recommended in patients with NAFLD and T2DM [13]. Indeed, there is some epidemiological data pointing to a reduced incidence of liver and non-liver malignancies including hepatocellular carcinoma [14].

\section{Glucagon-Like Peptide-1 (Agonists)}

Glucagon-like peptide-1 (GLP-1) is a gut-derived hormone analogue inducing insulin secretion, reducing glucagon secretion, suppressing appetite, and delaying gastric emptying [15]. GLP-1 analogues have been shown to improve liver histology in murine models of NASH [16, 17].

Liraglutide, as a long-acting GLP-1 analogue induced improvements in peripheral, hepatic and adipose insulin resistance. Moreover, it reduced de novo lipogenesis in patients with NASH [18]. After a one-year treatment, remarkable benefit on steatohepatitis was observed [19].

NAFLD/NASH: Successful Development

\section{De Novo Lipogenesis}

Aramchol is a conjugate of arachidic and cholic acid. In preclinical studies, aramchol reduced de novo lipogenesis $[21,22]$. NDI-010976 is an Acetyl-Co-A carboxylase inhibitor that was associated with increased fatty acid oxidation, reduced lipogenesis and hepatic fat content, and improved insulin sensitivity in rats [23]. Additionally, in obese adults, it was able to reduce de novo lipogenesis [24].

\section{Liver-Specific Targets}

For risk factors and processes affecting the liver itself, liver-specific therapy targets include fibrosis, hepatic fat accumulation and resultant metabolic stress, reducing oxidative stress and by this inflammation and injury, and influencing gut microbiota (Fig. 2).

\section{Vitamin E}

Vitamin E has been studied in Phase 2A and 2B trials [25-27]. Though, it was able to improve steatosis and steatohepatitis, it has no effect on fibrosis [1]. Unfortunately, there is some evidence for an increased risk to develop prostate cancer as well as bleeding incidents $[28,29]$. 
Fig. 2. Overview of the multimodal therapeutic strategies in NAFLD. Apart from the therapeutic options in the sector of weight loss, which have proven to be somewhat effective, all pharmaceutical options are still considered study medication or are in experimental stages. Especially in the field of targeting hepatic changes, many agents still need to be proven as relevant in NAFLD therapy in humans.

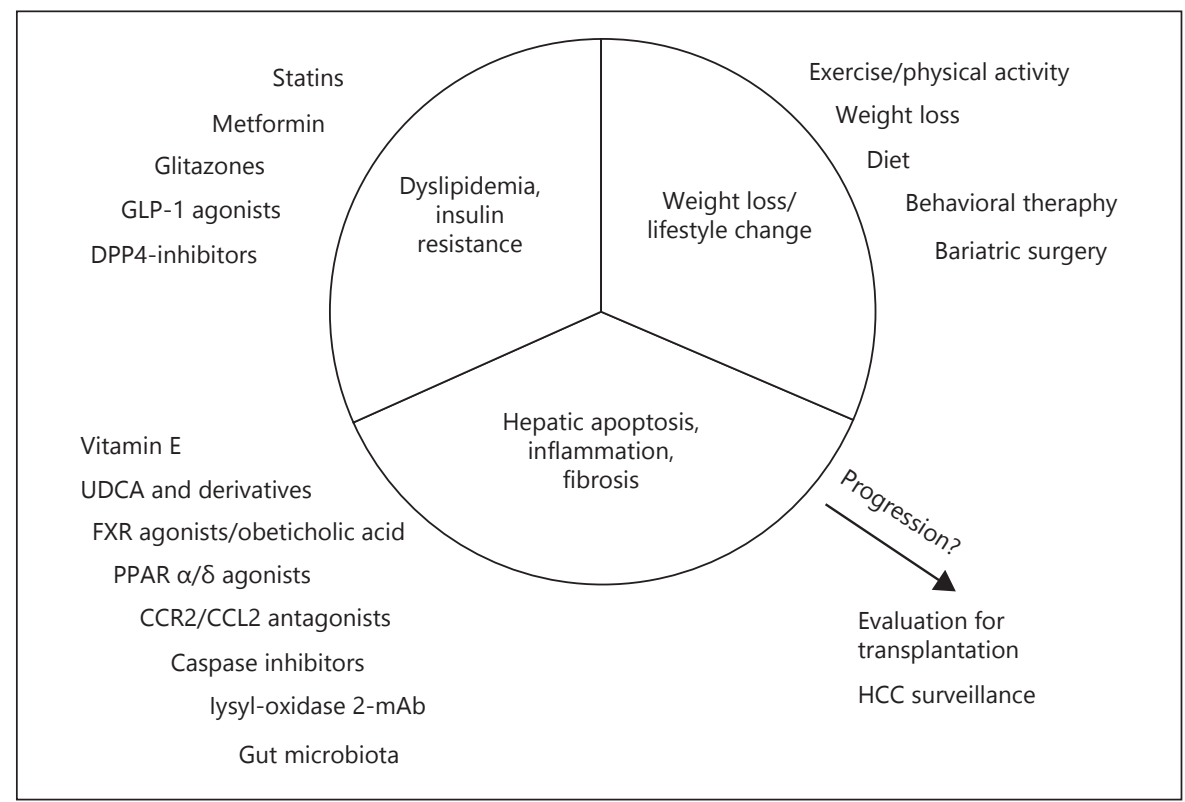

Ursodeoxycholic Acid and Derivatives

Ursodeoxycholic acid (UDCA), a naturally occurring secondary bile acid, has not been shown to improve histological features of NASH [30, 31]. Nevertheless, it reduces liver enzymes and improves markers of insulin sensitivity $[31,32]$. Interestingly, UDCA levels are per se higher in individuals with NAFLD and NASH even without UDCA treatment. This was found because of a changed secondary to primary bile acid ratio [33]. Unfortunately, there are no data supporting derivatives of UDCA in the treatment of NALFD/NASH, yet.

\section{The Farnesoid X Receptor-Bile Acid Axis}

FXR is expressed in liver, intestines, and kidneys and it plays a key role in bile acid synthesis [34]. Farnesoid X receptor (FXR) agonists such as GS-9674 reduced serum transaminases, hepatic steatosis and fibrosis in NASH.

Obeticholic acid (OCA) is a synthetic bile acid, which is able to improve NAS and fibrosis [35]. Indeed, OCA has been successfully used in the treatment of primary biliary cirrhosis. Sevelamer, a bile acid sequestrant, reduced steatosis and lobular inflammation in mice [36].

Peroxisome Proliferator-Activated Receptor $\gamma$-Agonist

Peroxisome proliferator-activated receptors $\gamma$ (PPARs) are well known to play a key role in the regulation of lipid metabolism and insulin sensitivity. One of the examples of this group is Pioglitazone. It improves NASH $[26,37,38]$ and in one meta-analysis was shown to improve hepatic fibrosis and insulin resistance [39].
However, it is also associated with weight gain and osteopenia [1]. Elafibranor (PPAR $\alpha \delta$ agonists) was able to improve steatosis, inflammation and fibrosis [40]. However, the treatment was associated with increased lipid oxidation and inhibited macrophage activation [41, 42]. Saroglitazar is a dual PPARa $\gamma$-agonist, which was associated with reduced steatosis, NASH and fibrosis in preclinical studies [43].

\section{Caspase Inhibitors}

Emricasan is an example of an irreversible pan-caspase inhibitor, which has been analyzed for pathways of inflammation and apoptosis in liver disease (Canbay JPET 2004). Indeed, the treatment reduced histological NAS and fibrosis scores in murine NAFLD models [44]. Current data in humans indicate a reduction in liver enzymes and markers of apoptosis (ck18 and M30). Though, it has to be considered that under continuous cell damage, as in ongoing NAFLD, caspase inhibition might not stop the disease but just lead to a switch in cell death mode. Whether this has beneficial or adverse effects remains to be seen. The role of other regulated cell death modes, that is, necroptosis, pyroptosis, ferroptosis, and if they offer possible therapeutic targets will have to be elucidated in a context that is relevant to humans.

\section{Lysyl Oxidase Like-2}

Lysyl oxidase like-2 (LOXL2) belongs to a family of enzymes involved in modifying the extracellular matrix, promoting cross-linking of cellular collagen and enhancing 
fibrosis [45]. Serum LOXL2 levels correlate with fibrosis in NAFLD [46]. Simtuzumab is a LOXL2 antibody, which improves fibrosis in combination with selonsertib [47].

\section{Anti-Inflammatory Agents}

While inflammation might be considered a consequence of the ongoing disease, reduction of the inflammatory component could possibly diminish further progression of NASH. Cenicriviroc, as a chemokine c-c-motif receptor (CCR)2-antagonist, did not meet the primary endpoint criteria of a 2-point reduction in NAS, but reduced fibrosis (secondary endpoint) in an RCT [48].

Pentoxifyllin as an anti-inflammatory agent improved $\mathrm{NASH}$ and decreased oxidized lipid products in small studies $[49,50]$.

\section{Upcoming Therapeutic Targets}

Gut Microbiota

Interaction of the liver and gut microbiome has been well documented in experimental studies. Targeting the gut microbiome is an overall promising approach to prevent hyperlipidemia. IMM-124e improved ALT and glucose tolerance in preclinical studies [51]. No clinical data for probiotic treatment are available as of right now, but a phase II RDBPCT is currently ongoing.

\section{Copper}

There is evidence that inadequate copper intake may be involved in the pathogenesis of NAFLD [52]. Not just in animals, but also in humans, alterations that link copper, mitochondrial function and NAFLD have been found [53]. Patients with M. Wilson, who accumulate copper in the liver, develop steatosis within the liver. Also, in $\mathrm{ATP} \mathrm{B}^{-1-}$ knockout mice (mouse model for Wilsons Disease), copper accumulation leads to steatosis and inflammation in hepatocytes $[54,55]$. Controlling copper transport and storage in the liver might be an attractive target to treat NAFLD.

\section{Summary}

NAFLD diagnosis should be performed in any patient with metabolic alterations (i.e., obesity or components of the metabolic syndrome present). To detect NASH, a liver biopsy is still required and noninvasive markers can only suggest a possible risk. Surveillance of NAFLD patients for cardiovascular disease (and outcomes), fibrosis but more importantly hepatocellular cancer (HCC) has to be improved dramatically.

NAFLD/NASH: Successful Development of Pharmacological Treatment
For conservative therapy of NAFLD or NASH, the first-line and mainstay should be weight loss by intense lifestyle change. Pharmacological options have not been approved yet, and comprise approaches targeting insulin resistance or dyslipidemia as underlying cause for NAFLD or are directed toward targets in the liver. Future studies will have to show, if any pharmacological treatment is truly effective against NAFLD or even NASH. Novel targets are the gut microbiome-liver axis and copper as rare element, as it might be involved in disturbed lipid metabolism. One important limitation of the search for therapeutic targets must be kept in mind: most of these potential targets derive from murine models with substantial difference in genetic background but also profound anatomical differences regarding the digestive system. Therefore, it is of utmost importance to conduct studies to identify possible therapeutic targets in humans. Targets derived from animal studies may be misleading in therapeutic success.

\section{Nonalcoholic Fatty Liver Disease: Treatment as Key Points}

- Nonalcoholic fatty liver disease: effective diet and lifestyle changes improve liver histology

- No specific therapy is currently approved for the treatment of NASH

- (No general recommendation for vitamin E, UDCA, silymarin, etc.)

- NAFLD: new treatment options are currently being evaluated, targeting insulin resistance, dyslipidemia and hepatic injury/inflammation/fibrosis

- Promising approaches are GLP1 agonists (weight reduction), OCA (FXR agonism), elafibranor (PPAR $\alpha / \delta$ agonist), and CCR2/CCR5 inhibitor cenicriviroc (anti-fibrotic)

- Surveillance for liver-related complications (cirrhosis, portal hypertension, HCC) and comorbidities is mandatory in high-risk patients

- Mechanisms of human and murine NAFLD/NASH differ substantially, and therefore, we need more studies targeting NALFD in humans

- Targeting copper might be an attractive target.

\section{Disclosure Statement}

The authors declare that they have no conflicts of interest to disclose. 


\section{References}

1 Banini BA, Sanyal AJ: Nonalcoholic fatty liver disease: epidemiology, pathogenesis, natural history, diagnosis, and current treatment options. Clin Med Insights Ther 2016;8:75-84.

2 Usluer G, Erben N, Aykin N, et al: Comparison of non-invasive fibrosis markers and classical liver biopsy in chronic hepatitis C. Eur J Clin Microbiol Infect Dis 2012;31:1873-1878.

3 Arora A, Sharma P: Non-invasive diagnosis of fibrosis in non-alcoholic fatty liver disease. J Clin Exp Hepatol 2012;2:145-155.

4 EASL-EASD-EASO Clinical Practice Guidelines for the Management of Non-Alcoholic Fatty Liver Disease. J Hepatol 2017;64:13881402.

5 VanWagner LB, Armstrong MJ: Lean NAFLD: a not so benign condition? Hepatol Commun 2018;2:5-8.

6 Teufel A, Itzel T, Erhart W, et al: Comparison of gene expression patterns between mouse models of nonalcoholic fatty liver disease and liver tissues from patients. Gastroenterology 2016; 151: 513-525.e0.

7 Vilar-Gomez E, Martinez-Perez Y, Calzadilla-Bertot L, et al: Weight loss through lifestyle modification significantly reduces features of nonalcoholic steatohepatitis. Gastroenterology 2015;149:367-78.e5; quiz e14-e15.

8 Chalasani N, Aljadhey H, Kesterson J, et al: Patients with elevated liver enzymes are not at higher risk for statin hepatotoxicity. Gastroenterology 2004;126:1287-1292.

9 Tziomalos K, Athyros VG, Paschos P, et al: Nonalcoholic fatty liver disease and statins. Metab Clin Exp 2015;64:1215-1223.

10 Lobo SM, Quinto BM, Oyama L, et al: TNF-a modulates statin effects on secretion and expression of MCP-1, PAI-1 and adiponectin in 3T3-L1 differentiated adipocytes. Cytokine 2012;60:150-156.

11 Abraldes JG, Albillos A, Bañares R, et al: Simvastatin lowers portal pressure in patients with cirrhosis and portal hypertension: a randomized controlled trial. Gastroenterology 2009;136:1651-1658.

12 Maruthur NM, Tseng E, Hutfless S, et al: Diabetes medications as monotherapy or metformin-based combination therapy for type 2 diabetes: a systematic review and meta-analysis. Ann Intern Med 2016;164:740-751.

13 Musso G, Cassader M, Rosina F, et al: Impact of current treatments on liver disease, glucose metabolism and cardiovascular risk in nonalcoholic fatty liver disease (NAFLD): a systematic review and meta-analysis of randomised trials. Diabetologia 2012;55:885904.

14 Chen HP, Shieh JJ, Chang CC, et al: Metformin decreases hepatocellular carcinoma risk in a dose-dependent manner: populationbased and in vitro studies. Gut 2013;62:606615.

15 Baggio LL, Drucker DJ: Biology of incretins: GLP-1 and GIP. Gastroenterology 2007;132: 2131-2157.
16 Ding X, Saxena NK, Lin S, et al: Exendin-4, a glucagon-like protein-1 (GLP-1) receptor agonist, reverses hepatic steatosis in ob/ob mice. Hepatology 2006;43:173-181.

17 Mells JE, Fu PP, Sharma S, et al: Glp-1 analog, liraglutide, ameliorates hepatic steatosis and cardiac hypertrophy in C57BL/6J mice fed a Western diet. Am J Physiol Gastrointest Liver Physiol 2012;302:G225-G235.

18 Armstrong MJ, Hull D, Guo K, et al: Glucagon-like peptide 1 decreases lipotoxicity in non-alcoholic steatohepatitis. J Hepatol 2016; 64:399-408.

19 Armstrong MJ, Gaunt P, Aithal GP, et al: Liraglutide safety and efficacy in patients with nonalcoholic steatohepatitis (LEAN): a multicentre, double-blind, randomised, placebo-controlled phase 2 study. Lancet 2016;387:679-690.

20 Yilmaz Y, Yonal O, Deyneli O, et al: Effects of sitagliptin in diabetic patients with nonalcoholic steatohepatitis. Acta Gastroenterol Belg 2012;75:240-244

21 Leikin-Frenkel A, Goldiner I, Leikin-Gobbi $\mathrm{D}$, et al: Treatment of preestablished diet-induced fatty liver by oral fatty acid-bile acid conjugates in rodents. Eur J Gastroenterol Hepatol 2008;20:1205-1213.

22 Leikin-Frenkel A, Parini P, Konikoff FM, et al: Hypocholesterolemic effects of fatty acid bile acid conjugates (FABACs) in mice. Arch Biochem Biophys 2008;471:63-71.

23 Foster DW, Malonyl CA: The regulator of fatty acid synthesis and oxidation. J Clin Invest 2012;122:1958-1959.

24 Stiede K, Miao W, Blanchette HS, et al: Acetyl-coenzyme A carboxylase inhibition reduces de novo lipogenesis in overweight male subjects: a randomized, double-blind, crossover study. Hepatology 2017;66:324-334.

25 Sato K, Gosho M, Yamamoto T, et al: Vitamin $\mathrm{E}$ has a beneficial effect on nonalcoholic fatty liver disease: a meta-analysis of randomized controlled trials. Nutrition 2015;3:923-930.

26 Sanyal AJ, Chalasani N, Kowdley KV, et al: Pioglitazone, vitamin E, or placebo for nonalcoholic steatohepatitis. N Engl J Med 2010; 362:1675-1685.

27 Lavine JE, Schwimmer JB, van Natta ML, et al: Effect of vitamin $\mathrm{E}$ or metformin for treatment of nonalcoholic fatty liver disease in children and adolescents: the TONIC randomized controlled trial. JAMA 2011;305: 1659-1668.

28 Yang XP, Lai D, Zhong XY, et al: Efficacy and safety of canagliflozin in subjects with type 2 diabetes: Systematic review and meta-analysis. Eur J Clin Pharmacol 2014;70:1149-1158.

29 Townsend SA, Newsome PN: Review article: new treatments in non-alcoholic fatty liver disease. Aliment Pharmacol Ther 2017;46: 494-507.

30 Liechti F, Dufour JF: Treatment of NASH with ursodeoxycholic acid: Cons. Clin Res Hepatol Gastroenterol 2012;36(suppl 1):S46S52.
31 Ratziu V: Treatment of NASH with ursodeoxycholic acid: Pro. Clin Res Hepatol Gastroenterol 2012;36(suppl 1):S41-S45.

32 Leuschner UF, Lindenthal B, Herrmann G, et al: High-dose ursodeoxycholic acid therapy for nonalcoholic steatohepatitis: a doubleblind, randomized, placebo-controlled trial. Hepatology 2010;52:472-479.

33 Puri P, Daita K, Joyce A, et al: The presence and severity of nonalcoholic steatohepatitis is associated with specific changes in circulating bile acids. Hepatology 2017, Epub ahead of print.

34 Bechmann LP, Kocabayoglu P, Sowa JP, et al: Free fatty acids repress small heterodimer partner (SHP) activation and adiponectin counteracts bile acid-induced liver injury in superobese patients with nonalcoholic steatohepatitis. Hepatology 2013;57:1394-1406.

35 Neuschwander-Tetri BA, Loomba R, Sanyal AJ, et al: Farnesoid X nuclear receptor ligand obeticholic acid for non-cirrhotic, non-alcoholic steatohepatitis (FLINT): a multicentre, randomised, placebo-controlled trial. Lancet 2015;385:956-965.

36 McGettigan BM, McMahan RH, Luo Y, et al Sevelamer improves steatohepatitis, inhibits liver and intestinal farnesoid X receptor (FXR), and reverses innate immune dysregulation in a mouse model of non-alcoholic fatty liver disease. J Biol Chem 2016;291:23058-23067.

37 Promrat K, Lutchman G, Uwaifo GI, et al: A pilot study of pioglitazone treatment for nonalcoholic steatohepatitis. Hepatology 2004; 39:188-196.

38 Belfort R, Harrison SA, Brown K, et al: A placebo-controlled trial of pioglitazone in subjects with nonalcoholic steatohepatitis. N Engl J Med 2006;355:2297-2307.

39 Singh S, Khera R, Allen AM, et al: Comparative effectiveness of pharmacological interventions for nonalcoholic steatohepatitis: a systematic review and network meta-analysis. Hepatology 2015;62:1417-1432.

40 Staels B, Rubenstrunk A, Noel B, et al: Hepatoprotective effects of the dual peroxisome proliferator-activated receptor alpha/delta agonist, GFT505, in rodent models of nonalcoholic fatty liver disease/nonalcoholic steatohepatitis. Hepatology 2013;58:1941-1952.

41 Risérus U, Sprecher D, Johnson T, et al: Activation of peroxisome proliferator-activated receptor (PPAR)delta promotes reversal of multiple metabolic abnormalities, reduces oxidative stress, and increases fatty acid oxidation in moderately obese men. Diabetes 2008;57:332-339.

42 Odegaard JI, Ricardo-Gonzalez RR, Red Eagle A, et al: Alternative M2 activation of Kupffer cells by PPARdelta ameliorates obesity-induced insulin resistance. Cell Metab 2008;7: 496-507.

43 Jain MR, Giri SR, Bhoi B, et al: Dual PPARa/ $\gamma$ agonist saroglitazar improves liver histopathology and biochemistry in experimental NASH models. Liver Int 2018;38:1084-1094. 
44 Barreyro FJ, Holod S, Finocchietto PV, et al: The pan-caspase inhibitor Emricasan (IDN6556) decreases liver injury and fibrosis in a murine model of non-alcoholic steatohepatitis. Liver Int 2015;35:953-966.

45 Moon HJ, Finney J, Ronnebaum T, et al: $\mathrm{Hu}-$ man lysyl oxidase-like 2. Bioorg Chem 2014; 57:231-241.

46 Murawaki Y, Kusakabe Y, Hirayama C: Serum lysyl oxidase activity in chronic liver disease in comparison with serum levels of prolyl hydroxylase and laminin. Hepatology 1991; 14:1167-1173.

47 Loomba R, Lawitz E, Mantry PS, et al: The ASK1 inhibitor selonsertib in patients with nonalcoholic steatohepatitis: a randomized, phase 2 trial. Hepatology 2017, Epub ahead of print.
48 Friedman SL, Ratziu V, Harrison SA et al. A randomized, placebo-controlled trial of cenicriviroc for treatment of nonalcoholic steatohepatitis with fibrosis. Hepatology 2018; 67:1754-1767.

49 Zein CO, Lopez R, Fu X, et al: Pentoxifylline decreases oxidized lipid products in nonalcoholic steatohepatitis: new evidence on the potential therapeutic mechanism. Hepatology 2012;56:1291-1299.

50 Zein CO, Yerian LM, Gogate P, et al: Pentoxifylline improves nonalcoholic steatohepatitis: A randomized placebo-controlled trial. Hepatology 2011;54:1610-1619.

51 Adar T, Ben Ya'acov A, Lalazar G, et al: Oral administration of immunoglobulin G-enhanced colostrum alleviates insulin resistance and liver injury and is associated with alterations in natural killer T cells. Clin Exp Immunol 2012;167:252-260.
52 Tallino S, Duffy M, Ralle M, et al: Nutrigenomics analysis reveals that copper deficiency and dietary sucrose up-regulate inflammation, fibrosis and lipogenic pathways in a mature rat model of nonalcoholic fatty liver disease. J Nutr Biochem 2015;26:996-1006.

53 Antonucci L, Porcu C, Iannucci G, et al: Nonalcoholic fatty liver disease and nutritional implications: special focus on copper. Nutrients 2017;9:pii:E1137.

54 Huster D: Structural and metabolic changes in Atp $7 \mathrm{~b}^{-/-}$mouse liver and potential for new interventions in Wilson's disease. Ann N Y Acad Sci 2014;1315:37-44.

55 Huster D, Kühne A, Bhattacharjee A, et al: Diverse functional properties of wilson disease ATP7B variants. Gastroenterology 2012;142: 947-956. 